\title{
Evaluación inicial de un programa de desarrollo valoral a nivel de educación básica en ciudad Juárez
}

\author{
Ricardo Almeida Uranga \\ Universidad Autónoma de Ciudad Juárez, \\ Instituto de Ciencias Sociales y Administración \\ ralmeida@uacj.mx \\ Oliverio Ismael Ferman Ávila \\ Programa de Educación en Valores de Ciudad Juárez AC \\ ismael.ferman@hotmail.com
}

\begin{abstract}
Resumen
Durante el ciclo escolar 2010-2011 el Programa de Educación en Valores (PEV) trabajó en 670 escuelas de educación básica en Juárez. Dicha cantidad representa el 11.3\% de escuelas del nivel básico en la entidad; y un $57.2 \%$ del total de escuelas de educación básica en el municipio de Juárez. La misión del PEV consiste en "garantizar la promoción de un cambio permanente en la comunidad educativa hacia mejores actitudes éticas" (PEV, pág. 5). Algunos de los ejes sobre los que se sustenta su misión son: (a) que la intervención sea preventiva; (b) que los programas sean propositivos; (c) que el trabajo sea centrado en las personas; (d) que el trabajo esté orientado a los docentes y directivos; (e) y que su enfoque sea holístico. Los trabajos del PEV se constituyen en una aportación valiosa para el desarrollo valoral y formación en ciudadanía que es recomendable documentar. Los propósitos del presente trabajo consisten primeramente en describir el "Modelo de Intervención del PEV" que se implementa en las escuelas de educación básica en el municipio de Juárez; posteriormente el siguiente objetivo es presentar los resultados de la evaluación que se realizó del PEV entre el 2008 y el 2010. Dicho estudio se realizó como un esfuerzo para valorar el impacto del trabajo de campo que realizan en las escuelas participantes. En este reporte se tratan dichos temas y se presentan también los resultados del estudio evaluativo del PEV, la interpretación de dichos datos y las conclusiones de manera muy sintética.
\end{abstract}

\section{Palabras clave}

Ambiente educativo, cambio educacional, clima escolar, ciudadanía, desarrollo de programas.

\section{Introducción}

Los propósitos del presente trabajo consisten en describir el "Modelo de Intervención del $P E V^{\prime \prime}$ que se implementa en las escuelas de educación básica en el municipio de Juárez; y presentar los resultados de una encuesta de evaluación inicial que se realizó desde el PEV entre el 2008 y el 2010. El estudio fue un esfuerzo para evaluar el trabajo de campo que se realizó en las escuelas participantes del PEV.

Caracterización breve del modelo de intervención del Programa de Educación en Valores

En Ciudad Juárez se lleva a cabo en la actualidad un programa de intervención 
en las escuelas del nivel básico que intenta propiciar una educación que, sin perder de vista la enseñanza de los contenidos curriculares oficiales, atienda también la dimensión del desarrollo valoral. El programa se denomina "Programa de Educación en Valores" (PEV) y fue fundado desde 1995 por un grupo de ciudadanos preocupados por la problemática del municipio. El PEV se fundó como una asociación civil: "sin fines de lucro que intenta coadyuvar a la formación integral del individuo con acciones dirigidas a la educación, conocimiento y práctica de valores éticos, como alternativa de solución a la crisis social, cultural y ciudadana que se presenta en el cotidiano devenir de esta sociedad fronteriza" (2008, pág. 4).

Durante el ciclo escolar 2010-2011 el PEV trabajó en 670 escuelas de educación básica en Juárez. Dicha cantidad representó el $11.3 \%$ de escuelas del nivel básico en la entidad; y un 57.2\% del total de escuelas de educación básica en el municipio de Juárez. La misión del PEV consiste en "garantizar la promoción de un cambio permanente en la comunidad hacia mejores actitudes éticas" (PEV, pág. 5).

La filosofía del PEV se sustenta en aquellos enfoques educativos que conciben a la escuela como el lugar por excelencia donde se aprende a convivir y se construyen socialmente los valores (Delors, 1996; Elexpuru y Garma, 2000; Guevara, 1992; Rojas y Lambrecht, 2000). Esta construcción se da al interior de la institución escolar a través del planteamiento de estrategias didácticas adecuadas y a través de un clima propicio que permite el ejercicio de la reflexión crítica y la consecuente toma de decisiones. Las investigaciones actuales en el campo de la educación valoral marcan dos vías en los procesos de construcción de valores (Cortina, 1997; Escámez, PérezDelgado, Domingo, Escrivá y Pérez, 1998; Hansen, 2002; Martínez, 2002; Martínez y Bujons, 2001; Martínez y Hoyos, 2004; Martínez, Tey y Campo, 2006):

I. La primera, orientada a la construcción de valores a través de la propia gestión de un currículo que se orienta a la transversalidad (ver Cuadro 1, en la sección de "Cuadros");

II. Y la segunda, la construcción de valores y actitudes mediante el propio proyecto de gestión escolar o proyecto institucional (ver Cuadro 2, en la sección de "Cuadros").

Ambas vías son imperativas, se relacionan entre sí y también se retroalimentan. Y es precisamente en esta última vía donde se habla de la construcción de un clima escolar propicio para estimular el desarrollo de actitudes y valores para la ciudadanía en un ámbito democrático. Estos conceptos han proporcionado la directriz teórica para las intervenciones del PEV en Juárez. Estos conceptos han proporcionado la directriz teórica para las intervenciones del PEV en Juárez.

Como aportación a la segunda vía, el PEV desarrolla en las escuelas de educación básica en Juárez el "Proyecto de Valores Éticos en la Escuela-PROVEE" (Ferman, 2009; Ferman y Almeida, 2010; PEV, 2011; PROVEE, ver Cuadro 3 en la sección de "Cuadros"). Se espera que el "Proyecto de Valores Éticos en la Escuela" (PROVEE) influya en las actitudes entre maestros, directivos, padres de familia y estudiantes, para que dichos grupos se sensibilicen al tema de valores. El PROVEE parte de la hipótesis de que toda institución escolar debe avalar su función social con un proyecto educativo que exprese el ideal de la calidad educativa al 
que se compromete y con el cual se responsabiliza ante la comunidad. En dicho proyecto se atienden dos dimensiones: (a) la dimensión académica o informativa; (b) y la dimensión ética o formativa (Ferman y Junco, 2006).

La intervención del PEV consiste en asesorar al colectivo escolar en la elaboración de la propuesta para la implementación del proyecto que le permita a la comunidad educativa sustentar una mayor calidad educativa incluyendo en sus planteamientos la práctica de valores ciudadanos.

\section{Cuestión central y diseño del sondeo de opinión}

Cuestión central

Surgió la inquietud de realizar una indagación inicial de la influencia de funcionamiento del PEV sobre la gestión escolar y el clima de relaciones en un grupo de las escuelas participantes. Por ello se planteó como pregunta central para guiar la presente investigación evaluativa la siguiente pregunta:

¿Qué opinan estudiantes, padres y maestros sobre la conducción del proyecto de gestión y del clima escolar en escuelas participantes del PEV, particularmente en las escuelas con niveles de implementación más avanzados; y cómo ha variado dicha opinión entre el 2009 y el 2010?

\section{Diseño de la encuesta para el levantamiento de información}

Dado el tamaño de la población meta, que en todos los casos se mide en miles de personas, se decidió que la técnica idónea para recabar los datos era la encuesta (Giroux y Tremblay, 2004; Hernández, Fernández-Collado y Baptista, 2006; Kerlinger y Howard, 2001; Ramírez y Murphy, 2007).

El diseño de la encuesta se llevó a cabo mediante un ejercicio de corte generativo durante el 2008. En este proceso participaron la Dirección Ejecutiva del PEV, el staff administrativo y el equipo de asesores con experiencia de campo del PEV. Asimismo, se invitaron a especialistas en educación externos para que guiaran y validaran desde su perspectiva como "expertos" el ejercicio de generación de categorías. Los pasos para el diseño de las encuestas que se siguieron se inspiraron en la metodología para la construcción de instrumentos propuesta por Ramírez (consultado en 2008; Ramírez y Murphy, 2007). Se elaboraron las categorías y los reactivos para el diseño de las encuestas para estudiantes, padres y docentes incluyendo una amplia revisión de la literatura del área (Camacho, 2001; Coordinación de Investigación y Desarrollo Académico, 1997; Delors, 1996; Elexpuru y Garma, 2000; Guevara, 1992; Cortina, 1994, 1997; Escámez, PérezDelgado, Domingo, Escrivá y Pérez, 1998; Hansen, 2002; Hargreaves, 1996; Kerlinger y Howard, 2001; Kohlberg, 1992; Martínez, 2002; Martínez y Bujons, 2001; Martínez y Hoyos, 2004; Martínez, Tey y Campo, 2006; Mugny y Perez, 1998; Novak y Gowin, 1988; Ortega, 2008; Piaget, 1984; Rodríguez, 2004; Secretaría de Educación Pública, 2001.

\section{Decisión sobre las muestras}

Se realizaron dos levantamientos de datos que se llevaron a cabo, uno en febrero de 2009 y el segundo en diciembre de 2010, mediante la aplicación de una encuesta de evaluación entre estudiantes, padres y docentes.

Las muestras seleccionadas fueron no probabilísticas por lo que no pretenden generalizarse los resultados obtenidos a la población completa de escuelas participantes del PEV. El criterio de selección de las escuelas fue que se incluyeran en la muestra de estudio las 
RECIE. Revista Electrónica Científica de Investigación Educativa Vol. 1, núm. 1, enero-diciembre 2012, pp. 251-260.

que estuvieran en la fase de implementación más avanzada del PROVEE con el objeto de que los estudiantes, padres y maestros ya tuviesen referentes de actividades y cambios realizados en la escuela como consecuencia de la intervención del PEV. La información que se presenta pretende constituirse en una evaluación inicial de la influencia que ha tenido el PEV en la gestión escolar y en la construcción de valores de las comunidades escolares en las que se ha estado interviniendo.

Para la conducción de la encuesta de evaluación en febrero de 2009 se determinó una muestra no probabilística de 40 escuelas a las que se aplicaron encuestas de entrada (pre-test); y en el de diciembre de 2010 se utilizó la misma muestra más dos escuelas adicionales quedando el total en 42 escuelas, a las que se aplicaron encuestas de salida (posttest).

En la Tabla 1 (ver la sección de "Tablas") se especifican los grupos y tamaños de las muestras del presente estudio.

\section{Procesamiento y análisis de datos}

Se generó una base de datos con la información de entrada correspondiente a febrero de 2009 y de salida correspondiente a diciembre de 2010, de acuerdo al rol de los participantes, en hojas de captura del "Paquete Estadístico para las Ciencias Sociales" (Gardner, 2003). Se estimaron las medias estadísticas por categoría y participantes. Posteriormente se estimaron los promedios de las medias obtenidas en el paso anterior para cada categoría y desagregado por el rol del respondiente del total de escuelas incluidas en la muestra. Posteriormente, se utilizó la $t$-student para determinar el nivel de significancia estadística de las diferencias entre las medias de cada grupo (aplicación de febrero 2009 versus aplicación de diciembre 2010).

\section{Interpretación de resultados y conclusión}

En base a la pregunta central e inicial se presentan las siguientes conclusiones (ver tablas 2 a la 4 en la sección de "Tablas").

Primeramente, destacar que la opinión en general, tanto de estudiantes como de padres y maestros tiende a ubicarse en promedio en el nivel de "satisfactorio" en relación a la gestión escolar que utiliza la herramienta de "planeación de proyecto educativo" y que es instrumentada a través del PROVEE. Dicho nivel de opinión tiende a ser "estable" en los dos periodos considerados de aplicación de la encuesta (febrero de 2009 y diciembre de 2010). En la misma opinión manifestada por las escuelas encuestadas se vislumbra una asociación inicial (y que requiere validarse con análisis ulteriores) entre la gestión escolar, el PROVEE y una mejoría en algunos aspectos o categorías del clima escolar en los que se toman en cuenta también actividades para propiciar la construcción de valores.

En general, las tendencias de opinión manifiestan mejoras en los diferentes rubros de la operación del programa educativo y de la propia escuela. Sin embargo, es interesante y contrastante hacer notar que dentro del grupo de padres de familia tiende a haber una opinión de menor satisfacción en las categorías que tienen que ver con el nivel de comunicación que mantienen con sus hijos(as), su propia participación en las actividades escolares y la opinión que tienen de la calidad de los procesos educativos que brinda la escuela. Si bien es cierto, que también los maestros pueden tener el rol en sus propias casas de 
"padres" y "madres", llama la atención que en el grupo de "padres de familia" específicamente haya en promedio menor satisfacción con aspectos que tienen que ver con la convivencia con sus hijos(as), con la propia "convivencia" con la escuela y con lo que se opina en relación a la calidad de la educación que proporciona la escuela.

Estos resultados nos invitan a reflexionar acerca de si el grupo de padres de familia de las escuelas participantes en el sondeo pudiesen ser quienes, como actores sociales, están siendo receptores y portadores de la mayor carga del "estrés social" derivado de la crisis económica y de inseguridad social que actualmente afectan a la población del municipio de Juárez. Si bien el diseño de la evaluación cuyos resultados se presentan aquí no permite el alcance de dichas inferencias, sí es importante de cualquier manera, dejar dicho argumento como un planteamiento hipotético para su posterior análisis $\mathrm{y}$ estudio.

Tablas

Tabla 1. Tamaños muestrales utilizados en el presente estudio.

\begin{tabular}{|l|l|l|l|}
\hline \multicolumn{1}{|c|}{ MUESTRAS } & \multicolumn{3}{|c|}{ Número de Encuestas } \\
\hline Fecha & Maestros & Padres & Alumnos \\
\hline Febrero 2009 & 304 & 1540 & 1080 \\
\hline Diciembre 2010 & 304 & 1153 & 958 \\
\hline Total & 608 & 2693 & 2038 \\
\hline
\end{tabular}

Tabla 2. Resultados para estudiantes.

\begin{tabular}{|c|c|c|c|c|c|c|}
\hline & \multirow{2}{*}{ No. } & \multirow{2}{*}{ Categoría } & \multicolumn{2}{|l|}{ Media } & \multirow[t]{2}{*}{ Dif. } & \multirow[t]{2}{*}{ Sig. } \\
\hline & & & $\begin{array}{l}\text { Febrero } \\
2009\end{array}$ & $\begin{array}{l}\text { Diciembre } \\
2010\end{array}$ & & \\
\hline \multirow{4}{*}{ 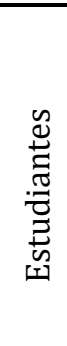 } & I & $\begin{array}{l}\text { Relaciones interpersonales entre los } \\
\text { maestros. }\end{array}$ & 4.46 & 4.53 & .065 & .057 \\
\hline & II & Utilización de recursos de apoyo. & 4.33 & 4.52 & .1894 & $.000^{*}$ \\
\hline & III & Relación de los alumnos con los padres. & 4.34 & 4.49 & .1516 & $.000^{*}$ \\
\hline & IV & Relación de los padres con la escuela. & 3.68 & 3.79 & .1334 & $.006^{*}$ \\
\hline
\end{tabular}

* Sig., son diferencias significativas a un nivel igual a 0.05; Dif. significa "diferencia aritmética"

Evaluación inicial de un programa de desarrollo valoral a nivel de educación básica... 
RECIE. Revista Electrónica Científica de Investigación Educativa Vol. 1, núm. 1, enero-diciembre 2012, pp. 251-260.

Tabla 3. Resultados para padres de familia.

\begin{tabular}{|c|c|c|c|c|c|c|}
\hline & \multirow[t]{2}{*}{ No. } & \multirow[t]{2}{*}{ Categoría } & \multicolumn{2}{|c|}{ Media } & \multirow[t]{2}{*}{ Dif. } & \multirow[t]{2}{*}{ Sig. } \\
\hline & & & $\begin{array}{c}\text { Febrero } \\
2009\end{array}$ & $\begin{array}{c}\text { Diciembre } \\
2010 \\
\end{array}$ & & \\
\hline \multirow{4}{*}{ 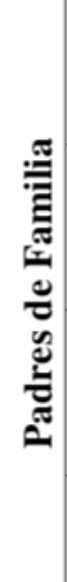 } & I & $\begin{array}{l}\text { Nivel de comunicación que mantiene } \\
\text { con su hijo(a) }\end{array}$ & 4.12 & 3.99 & .1250 & $.003 *$ \\
\hline & II & $\begin{array}{l}\text { Participación en las actividades que la } \\
\text { escuela convoca }\end{array}$ & 4.01 & 3.84 & .1665 & $.000^{*}$ \\
\hline & III & $\begin{array}{l}\text { Calidad de los procesos educativos } \\
\text { que brinda la escuela }\end{array}$ & 4.16 & 3.98 & $\begin{array}{c}- \\
.1469\end{array}$ & $.001 *$ \\
\hline & IV & $\begin{array}{l}\text { Apoyo específico a los hijos en la } \\
\text { realización de tareas escolares. }\end{array}$ & 4.50 & 4.53 & .0235 & .585 \\
\hline
\end{tabular}

* Sig., son diferencias significativas a un nivel igual a 0.05; Dif. significa "diferencia aritmética"

Tabla 4. Resultados para maestros.

\begin{tabular}{|c|c|c|c|c|c|c|}
\hline & \multirow[t]{2}{*}{ No. } & \multirow[t]{2}{*}{ Categoría } & \multicolumn{2}{|c|}{ Media } & \multirow[t]{2}{*}{ Dif. } & \multirow[t]{2}{*}{ Sig. } \\
\hline & & & $\begin{array}{c}\text { Febrero } \\
2009 \\
\end{array}$ & $\begin{array}{c}\text { Diciembre } \\
2010 \\
\end{array}$ & & \\
\hline \multirow{6}{*}{ 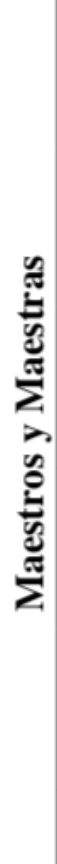 } & I & $\begin{array}{l}\text { Procesos de comunicación e } \\
\text { información al interior del colectivo } \\
\text { escolar. }\end{array}$ & 3.94 & 4.18 & .2957 & $.003 *$ \\
\hline & II & $\begin{array}{l}\text { Procesos de formación y desarrollo de } \\
\text { las habilidades docentes al interior del } \\
\text { colectivo escolar. }\end{array}$ & 3.88 & 3.89 & .0608 & .352 \\
\hline & III & $\begin{array}{l}\text { Establecimiento y cumplimiento de } \\
\text { objetivos en el marco del Proyecto } \\
\text { Escolar. }\end{array}$ & 4.06 & 4.10 & .0954 & .068 \\
\hline & IV & $\begin{array}{l}\text { Percepción sobre el } \\
\text { profesional y estímulos } \\
\text { desarrollo profesional. }\end{array}$ & 3.73 & 3.84 & .1971 & $.020^{*}$ \\
\hline & $\mathbf{V}$ & $\begin{array}{l}\text { Conocimiento y apego a la Misión, } \\
\text { Visión y Valores que la escuela busca a } \\
\text { través del Proyecto Escolar. }\end{array}$ & 4.05 & 4.08 & .0394 & .612 \\
\hline & VI & Calidad de las relaciones de trabajo. & 3.87 & 3.98 & .1525 & .062 \\
\hline
\end{tabular}

* Sig., son diferencias significativas a un nivel igual a 0.05; Dif. significa "diferencia aritmética" 
Cuadros Cuadro 1. Currículo transversal (Ferman y Junco, 2006)

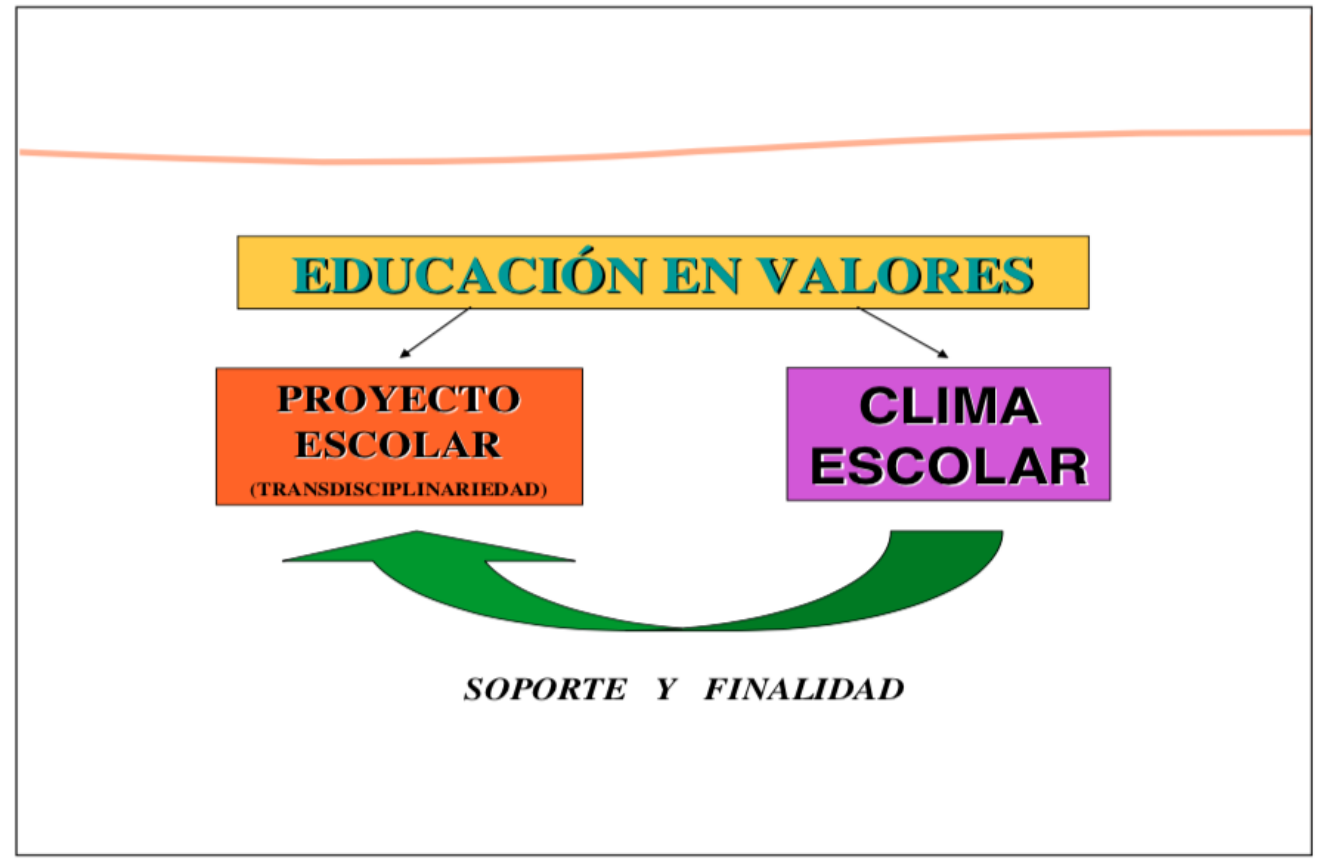

Cuadro 2. Proyecto informativo y formativo (Ferman y Junco, 2006).

$$
\text { TESIS FUNDAMENTAL }
$$

\section{INSTITUCION ESCOLAR}

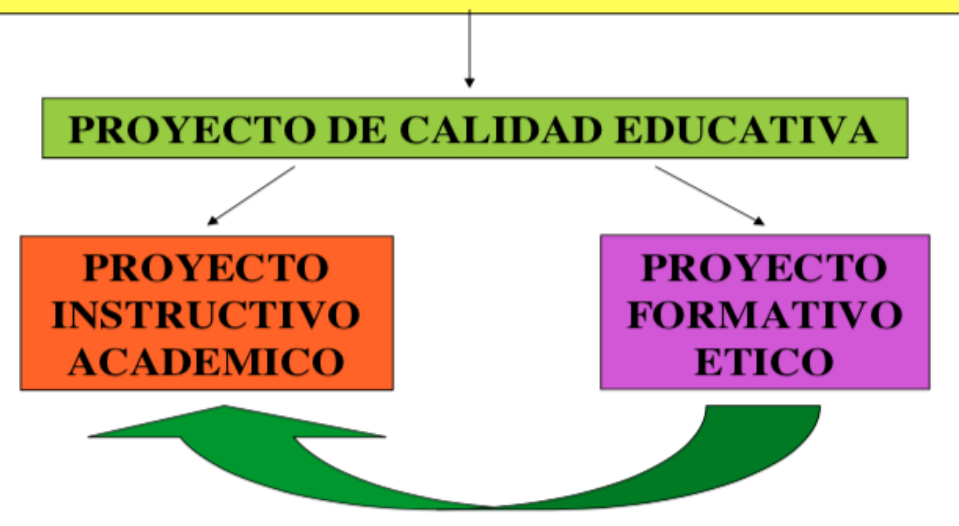

SOPORTE Y FINALIDAD

Evaluación inicial de un programa de desarrollo valoral a nivel de educación básica... 
Cuadro 3. Modelo general de intervención del PEV.

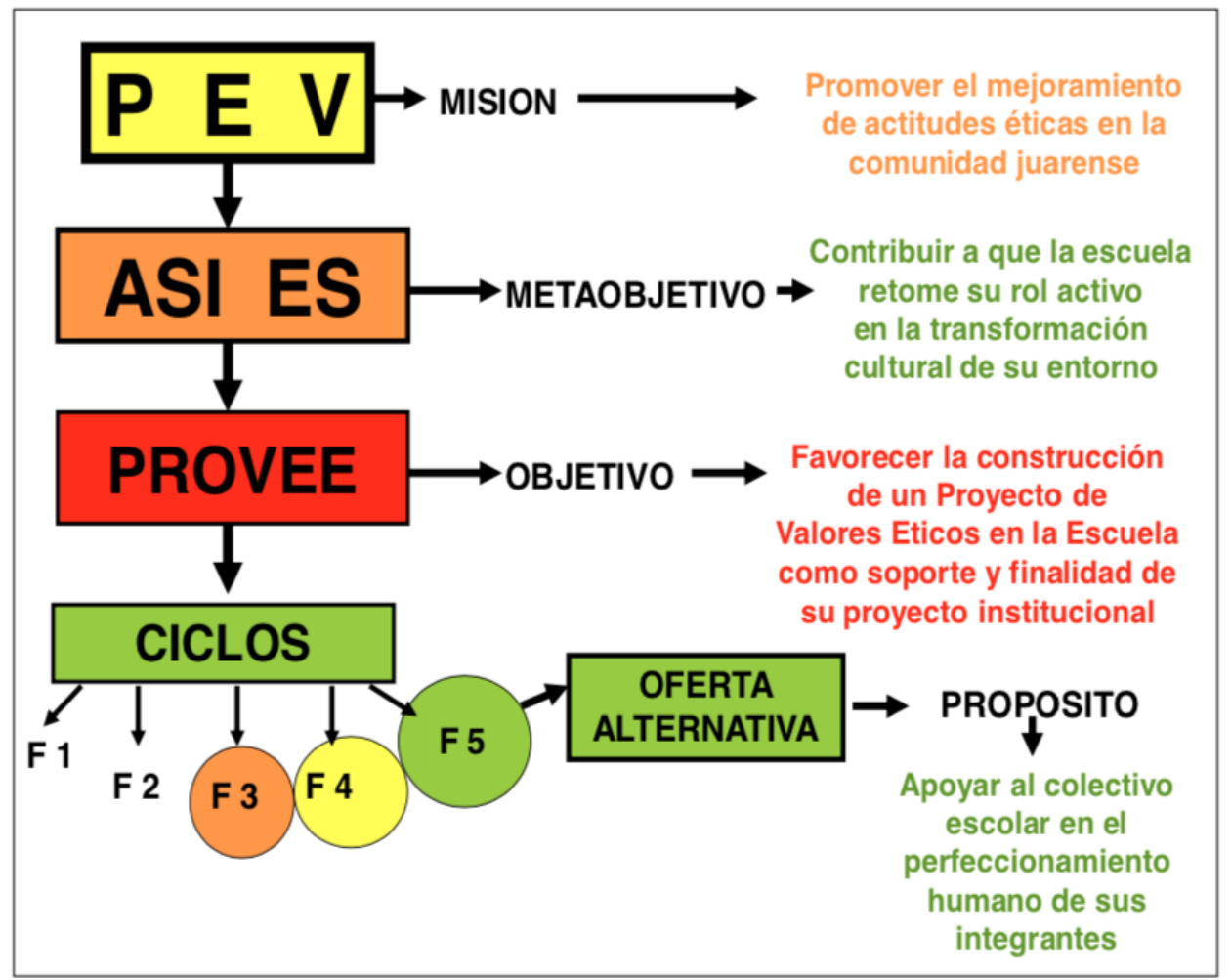

\section{Agradecimientos}

Se agradece el apoyo recibido por el Programa de Educación en Valores de Ciudad Juárez, A. C. para la realización del presente estudio.

\section{Referencias}

Camacho, S. (septiembre-diciembre 2001). Hacia una evaluación de la modernización educativa. Desarrollo y resultados del ANME". Revista Mexicana de Investigación Educativa, 6 (13), 401-423.

Coordinación de Investigación y Desarrollo Académico. Diagnóstico y Propuesta Educativa en Chihuahua. (octubre 1997). Foro 21. Reflexión, debate y propuesta educativa, II (10), 1.

Cortina, A. (1997). Ciudadanos del mundo: hacia una teoría de la ciudadanía. Madrid: Alianza Editorial.
Cortina, A. (1994). 10 palabras clave en ética. Navarra: Verbo Divino. Delors, J. (Chairman) (1996). Learning: The Treasure Within. París: UNESCO.

Elexpuru, I. y Garma, A. M. (2000). El autoconcepto en el aula, recursos para el profesorado. Barcelona: Edebe.

Escámez, J., Pérez-Delgado, E., Domingo, A., Escrivá, V. y Pérez, C. (1998). Educar en la autonomía moral. Valencia: Generalitat Valenciana, Consellería de Educación, Cultura y Ciencia.

Ferman, O. I. Evaluación de impacto en escuelas que desarrollan el proyecto de valores éticos (PROVEE) con el mayor nivel de implementación en primer y segundo ciclos de mejora continua (protocolo de investigación). Juárez, Chihuahua: 
Dirección Ejecutiva del PEV. Obtenida el 15 de enero de 2011 de http://www.educacionyvaloresj.or $\mathrm{g} /$

Ferman, O. I. y Junco, R. Modelo Educativo del Programa Educación en Valores. Juárez, Chihuahua: Dirección Ejecutiva del PEV. Obtenida el 15 de enero de 2011 de http://www.educacionyvaloresj.or g/

Ferman, O. I. y Almeida, R. Un estudio longitudinal: evaluación de impacto en escuelas que desarrollan el proyecto de valores éticos (PROVEE) con el mayor nivel de implementación en primer $y$ segundo ciclos de mejora continua. Juárez, Chihuahua: Dirección Ejecutiva del PEV. Obtenida el 15 de enero de 2011 de http://www.educacionyvaloresj.or g/

Gardner, R. C. (2003). Estadistica para Psicología Usando SPSS para Windows. Mexico: Pearson Educación, Prentice Hall.

Guevara, G. (Compilador) (1992). La catástrofe silenciosa. México: Fondo de Cultura Económica.

Giroux, S. y Tremblay, G. (2004). Metodología de las ciencias humanas. México: Fondo de Cultura Económica.

Gobierno Federal. (1993). Reforma del Artículo $3 o$ Constitucional y Ley General de Educación. México: SEP.

Hansen, D. T. (2002). Explorando el corazón moral de la enseñanza. Barcelona: Idea Books. Hargreaves, A. (1996). Profesorado, cultura y postmodernidad: cambian los tiempos, cambia el

profesorado. Madrid: Morata. Hernández, R., Fernández-Collado, C. y Baptista, P. (2006). Metodología de la
Investigación.

México: Mc Graw Hill. Instituto Nacional de Estadística y Geografía. (2011). Principales Resultados del Censo de

Población y Vivienda 2010. México: INEGI. Kerlinger, F. y Howard, L. (2001). Investigación del comportamiento: Métodos de

investigación en las Ciencias Sociales (4a). ed. México: Mc Graw Hill. Martínez, M. (2000). El contrato moral del profesorado. Condiciones para una nueva escuela.

Bilbao: Desclée De Brouwer. Martínez, M. y Bujons, C. (Coordinadores). (2001). Un lugar llamado escuela: en la sociedad de

la información $y$ de la diversidad. Barcelona: Ariel. Martínez, M. y Hoyos, G. (Coordinadores). (2004). ¿Qué significa educar en valores hoy?.

Barcelona: Octaedro-Organización de Estados

Iberoamericanos. Martínez, M., Tey, A. y Campo. A. (2006). La educación en valores y el aprendizaje ético en Por

preguntar que no quede. Madrid: Ministerio de Educación $\mathrm{y}$ Ciencia. Mugny, G. y Perez, J. A. (Coordinadores). (1998). Psicología social del desarrollo cognitivo.

Barcelona: Anthropos.

Musgrave, P. W. (1999). The Sociology of Education $\left(3^{\text {rd }}\right.$ ed.). London: Methuen.

Novak, J. D. y Gowin, D. B. (1988). Aprendiendo a aprender. Barcelona: Martínez Roca.

Ornelas, C. (1995). El Sistema Educativo Mexicano. México: Centro de Investigación y Docencia Económicas, Nacional Financiera, 
Fondo de Cultura Económica.

Ortega, F. J. (2008). Tendencias en la gestión de centros educativos en Revista Latinoamericana de Estudios Educativos. XXXVIII (1-2), 61-79.

Piaget, J. (1984). El criterio moral en el niño. Barcelona, Martínez Roca. Programa Educación en Valores. (2011). Manual de Procedimientos PROVEE. Juárez,

Chihuahua: Programa Educación en Valores (documento inédito de uso interno).

Programa Educación en Valores. (2008). Modelo de Intervención. Juárez, Chihuahua: Programa Educación en Valores

Ramírez, M.S.. "Construcción de Instrumentos". Monterrey, Nuevo León: Escuela de Graduados en Educación ITESM. Obtenida el 1 de junio de 2008, de http://videouv.itesm.mx/ege/ed5 004/irpe02/index.htm/

Ramírez, M. S. y Murphy, M. A. (Coordinadoras). (2007). Educación e investigación. Retos y oportunidades. México: Trillas.

Rodríguez, N. (2004). El clima escolar.
Investigación y Educación. 3 (7). Obtenido el 15 de febrero del 2009, en

http://www.csicsif.es/andalucia/ modules/

Rojas, A. y Lambrecht, N. (2000). Construyendo autoridad moral desde las escuelas. Reflexiones y propuestas para la acción. OREALC/UNESCO Santiago: UNESCO.

Secretaría de Educación Pública. (2001). ¿Cómo transformar las escuelas?. Lecciones desde la gestión escolar y la práctica pedagógica. Resultados del segundo estudio/diplomado de la vertiente de seguimiento del Estudio de Evaluación de la Educación Primaria. Resumen.

México: Sistema Educativo de los Estados Unidos Mexicanos.

Secretaría de Educación Pública. Principales cifras educativas para el ciclo escolar 2008-2009. México: Sistema Educativo de los Estados Unidos Mexicanos. Obtenida el 28 de marzo de 2011, en http://www.dgpp.sep.gob.mx/prin cipalescifras/ 